\title{
Arithmetic properties derived from coefficients of certain eta quotients
}

Jihyun Hwang ${ }^{1}$, Yan Li and Daeyeoul Kim ${ }^{3 *}$

\section{"Correspondence:}

kdaeyeoul@jbnu.ac.kr

${ }^{3}$ Department of Mathematics and Institute of Pure and Applied

Mathematics, Jeonbuk National University, Jeonju, South Korea Full list of author information is available at the end of the article

\section{Abstract}

For a positive integer $k$, let

$$
F(q)^{k}:=\prod_{n \geq 1} \frac{\left(1-q^{n}\right)^{4 k}}{\left(1+q^{2 n}\right)^{2 k}}=\sum_{n \geq 0} \mathfrak{a}_{k}(n) q^{n}
$$

be the eta quotients. The coefficients $\mathfrak{a}_{1}(n)$ can be interpreted as a certain kind of restricted divisor sums. In this paper, we give the signs and modulo values for $\mathfrak{a}_{1}(n)$ and $\mathfrak{a}_{2}(m)$ and calculate several convolution sums involving $\mathfrak{a}_{k}(n)$.

MSC: 11A07; 11A25;05A30

Keywords: Restricted divisor functions; Eta quotient; Convolution sums; q-series

\section{Introduction}

The study of arithmetical congruences is classical in elementary number theory, and such investigations have been carried out by several mathematicians including Ramanujan and Glaisher. For $d, m, n \in \mathbb{N}$ and $r, s \in \mathbb{N} \cup\{0\}$, we define some divisor functions for our further use:

$$
\sigma_{s}(n):=\sum_{d \mid n} d^{s}, \quad \bar{\sigma}(n):=\sum_{\substack{d \mid n \\ d \equiv \frac{n}{d}(\bmod 4)}} d-\sum_{\substack{d \mid n \\ d-\frac{n}{d} \equiv 2(\bmod 4)}} d, \quad \sigma_{s, r}(n ; m):=\sum_{\substack{d \mid n \\ d \equiv r(\bmod m)}} d^{s} .
$$

We also use the following convention:

$$
\sigma_{s}(n):=0 \quad \text { if } n \notin \mathbb{Z} \text { or } n<0, \quad \sigma(n):=\sigma_{1}(n)=\sum_{d \mid n} d
$$

The exact evaluation of the basic convolution sum

$$
\sum_{k=1}^{n-1} \sigma_{1}(k) \sigma_{1}(n-k)
$$

(c) The Author(s) 2020. This article is licensed under a Creative Commons Attribution 4.0 International License, which permits use, sharing, adaptation, distribution and reproduction in any medium or format, as long as you give appropriate credit to the original author(s) and the source, provide a link to the Creative Commons licence, and indicate if changes were made. The images or other third party material in this article are included in the article's Creative Commons licence, unless indicated otherwise in a credit line to the material. If material is not included in the article's Creative Commons licence and your intended use is not permitted by statutory regulation or exceeds the permitted use, you will need to obtain permission directly from the copyright holder. To view a copy of this licence, visit http://creativecommons.org/licenses/by/4.0/. 
first appeared in the letter of Besge to Liouville in 1862 [1]. The evaluation of such sums also appear in the works of Glaisher [5], Lahiri [11], Lehmer [12], Ramanujan [18], Skoruppa [19], and Williams [20]. For instance, Ramanujan obtained the identities

$$
\begin{aligned}
& \sum_{k=1}^{n-1} \sigma_{1}(k) \sigma_{1}(n-k)=\frac{1}{12}\left(5 \sigma_{3}(n)+(1-6 n) \sigma(n)\right), \\
& \sum_{k=1}^{n-1} \sigma_{1}(k) \sigma_{3}(n-k)=\frac{1}{240}\left(21 \sigma_{5}(n)+(10-30 n) \sigma_{3}(n)-\sigma_{1}(n)\right),
\end{aligned}
$$

and

$$
\sum_{k=1}^{n-1} \sigma_{3}(k) \sigma_{3}(n-k)=\frac{1}{120}\left(\sigma_{7}(n)-\sigma_{3}(n)\right)
$$

using only elementary arguments. For $a, b, n \in \mathbb{N}$, Ramanujan showed that the sum

$$
S_{a, b}(n):=\sum_{k=1}^{n-1} \sigma_{a}(k) \sigma_{b}(n-k)
$$

can be evaluated in terms of the quantities

$$
\sigma_{a+b+1}(n), \quad \sigma_{a+b-1}(n), \quad \ldots, \quad \sigma_{3}(n), \quad \sigma_{1}(n)
$$

for the nine pairs $(a, b) \in \mathbb{N}^{2}$ satisfying

$$
a+b=2,4,6,8,12, \quad a \leq b, a \equiv b \equiv 1(\bmod 2) .
$$

Let

$$
\begin{aligned}
& F(q)^{k}:=\prod_{n \geq 1} \frac{\left(1-q^{n}\right)^{4 k}}{\left(1+q^{2 n}\right)^{2 k}}=\sum_{m \geq 0} \mathfrak{a}_{k}(m) q^{m}, \\
& G(q):=q \prod_{n \geq 1}\left(1-q^{2 n}\right)^{12}=\sum_{m \geq 0} \mathfrak{c}(m) q^{m}, \\
& H(q):=q \prod_{n \geq 1}\left(1-q^{n}\right)^{8}\left(1-q^{2 n}\right)^{8}=\sum_{m \geq 0} \mathfrak{d}(m) q^{m},
\end{aligned}
$$

and

$$
Y(q)^{k}:=q^{k} \prod_{n \geq 1}\left(1-q^{2 n}\right)^{4 k}\left(1-q^{4 n}\right)^{4 k}=\sum_{m \geq 0} \mathfrak{e}_{k}(m) q^{m} .
$$

Here $q$ denotes a fixed complex number with $|q|<1$, so that we may write $q=e^{\pi i \tau}$, where $\operatorname{Im}(\tau)>0$. For $k=2$, the right side of (4) becomes

$$
\left(\sum_{n \geq 0} \mathfrak{a}_{1}(n) q^{n}\right) \cdot\left(\sum_{m \geq 0} \mathfrak{a}_{1}(m) q^{m}\right)=\sum_{n \geq 0} \sum_{k=0}^{n} \mathfrak{a}_{1}(k) \mathfrak{a}_{1}(n-k) q^{n} .
$$


So we note that

$$
\sum_{n \geq 0} \mathfrak{a}_{2}(n) q^{n}=\sum_{n \geq 0} \sum_{k=0}^{n} \mathfrak{a}_{1}(k) \mathfrak{a}_{1}(n-k) q^{n} .
$$

More precisely, we prove the following theorems.

Theorem 1 Let $n=2^{a} m$ be a positive integer with $(2, m)=1$. Then

$$
\mathfrak{a}_{1}(n) \begin{cases}<0 & \text { if } n \equiv 1(\bmod 4) \text { or } a=2, \\ =0 & \text { if } n \equiv 2(\bmod 4) \\ >0 & \text { if } n \equiv 3(\bmod 4) \text { or } a \geq 3 .\end{cases}
$$

In particular, we get:

(1) If $n \equiv 3(\bmod 4)$, then $\mathfrak{a}_{1}(n) \equiv 0(\bmod 16)$.

(2) If $n \equiv 1(\bmod 4)$, then

$$
\mathfrak{a}_{1}(n) \equiv \begin{cases}4(\bmod 8) & \text { if } n \text { is square } \\ 0(\bmod 8) & \text { otherwise }\end{cases}
$$

(3) If $a \geq 2$, then

$$
\mathfrak{a}_{1}(n) \equiv \begin{cases}8(\bmod 16) & \text { if } a=2 \text { and } n \text { is square, } \\ 0(\bmod 16) & \text { if } a=2 \text { and } n \text { is non-square, } \\ 24(\bmod 48) & \text { if } a>2 \text { and } n \text { is square, } \\ 0(\bmod 48) & \text { otherwise. }\end{cases}
$$

Remark 1 Using computer program, L. Pehlivan and K.S. Williams found exact formula for $\mathfrak{a}_{1}(n)$ in [17, Theorem 1.2 (ii)] and [21, Table 1]. On the other hand, we will prove Theorem 1 using basic arithmetic tools.

Theorem 2 Let $2 n=2^{k} m$ be an even positive integer with $(2, m)=1$. Then

$$
\mathfrak{a}_{2}(2 n) \begin{cases}<0 & \text { if } 2 n \equiv 4(\bmod 8) \\ >0 & \text { otherwise }\end{cases}
$$

and $\mathfrak{a}_{2}(2 n) \equiv 0\left(\bmod 16 \sigma_{3}(m)\right)$. In particular, $\mathfrak{a}_{2}(4 l+2)=16 \sigma_{3}(2 l+1), \mathfrak{a}_{2}(8 l+4)=$ $-144 \sigma_{3}(2 l+1)$, and $\mathfrak{a}_{2}(8 l)=-16\left(\sigma_{3}(2 l)-25 \sigma_{3}(l)\right)$ for $l \in \mathbb{N}$.

Theorem 3 Let $m$ and $r$ be positive integers. Then

$$
\sum_{\substack{a_{1}+a_{2}+\cdots+a_{r}=m \\ a_{1}, a_{2}, \ldots, a_{r} \text { odd } \\ 0 \leq t_{i} \leq a_{i}}}\left(-\frac{1}{4}\right)^{\epsilon\left(t_{1}, a_{1}-t_{1}, \ldots, t_{r}, a_{r}-t_{r}\right)} \bar{\sigma}\left(t_{1}\right) \bar{\sigma}\left(a_{1}-t_{1}\right) \cdots \bar{\sigma}\left(t_{r}\right) \bar{\sigma}\left(a_{r}-t_{r}\right)=\left(-\frac{1}{2}\right)^{r} \mathfrak{e}_{r}(m) .
$$

Here $\epsilon\left(t_{1}, a_{1}-t_{1}, \ldots, t_{r}, a_{r}-t_{r}\right):=\#\left\{t_{i}=0\right.$ or $\left.a_{j}-t_{j}=0 \mid 1 \leq i, j \leq r\right\}$ and $\bar{\sigma}(0):=1$. 
Similarly, we get

$$
\sum_{\substack{a_{1}+a_{2}+\cdots+a_{r}=m \\ a_{1}, a_{2}, \ldots, a_{r} \text { odd }}} \prod_{i=1}^{r}\left(\bar{\sigma}\left(a_{i}\right)-2 \sum_{\substack{t_{i}=1 \\ a_{i} \neq 1}}^{a_{i}-1} \bar{\sigma}\left(t_{i}\right) \bar{\sigma}\left(a_{i}-t_{i}\right)\right)=\mathfrak{e}_{r}(m) .
$$

Using the theory of modular forms, we can also get several convolution sums.

Theorem 4 Let $n \geq 2$ be an integer. Then we have

$$
\sum_{k=1}^{n-1} \bar{\sigma}(k) \bar{\sigma}(n-k)=\sigma_{3}\left(\frac{n}{2}\right)-18 \sigma_{3}\left(\frac{n}{4}\right)+32 \sigma_{3}\left(\frac{n}{8}\right)+\frac{1}{2} \bar{\sigma}(n)-\frac{1}{2} \mathfrak{e}_{1}(n)
$$

In particular, if $4 \nmid n$, then

$$
\sum_{k=1}^{n-1} \bar{\sigma}(k) \bar{\sigma}(n-k)=\sigma_{3}\left(\frac{n}{2}\right)+\frac{1}{2} \chi(n) \sigma(n)-\frac{1}{2} \mathfrak{e}_{1}(n)
$$

where $\chi$ is the nontrivial Dirichlet character modulus 4.

To state the next theorems, for $i=1,2, \ldots, 7$, let

$$
q^{i} \prod_{n=1}^{\infty} \frac{\left(1-q^{n}\right)^{14-2 i}\left(1-q^{4 n}\right)^{6}\left(1-q^{16 n}\right)^{2 i-2}}{\left(1-q^{2 n}\right)^{7-i}\left(1-q^{8 n}\right)^{i-1}}=\sum_{n=1}^{\infty} t_{i}(n) q^{n}
$$

and put

$$
T(n):=\frac{49}{256} t_{1}(n)+\frac{99}{64} t_{2}(n)+\frac{105}{32} t_{3}(n)+\frac{5}{4} t_{4}(n)+\frac{15}{4} t_{5}(n)+6 t_{6}(n)+4 t_{7}(n) .
$$

Theorem 5 Let $n \geq 3$ be an integer. Then we have

$$
\begin{aligned}
& \sum_{\substack{a_{1}+a_{2}+a_{3}=n \\
a_{1}, a_{2}, a_{3} \geq 1}} \bar{\sigma}\left(a_{1}\right) \bar{\sigma}\left(a_{2}\right) \bar{\sigma}\left(a_{3}\right) \\
& = \begin{cases}(-1)^{\frac{n+1}{2}}\left(\frac{1}{256} \sigma_{5}(n)-\frac{3}{16} \sigma(n)\right)+T(n)-\frac{3}{8} \mathfrak{e}_{1}(n), & \text { if } n \text { is odd }, \\
\frac{3}{4} \sigma_{3}\left(\frac{n}{2}\right)+T(n), & \text { if } n \equiv 2(\bmod 4), \\
\frac{1}{8} \sigma_{5}\left(\frac{n}{4}\right)+\frac{3}{4} \sigma_{3}\left(\frac{n}{2}\right)-\frac{27}{2} \sigma_{3}\left(\frac{n}{4}\right)+\frac{3}{8} \sigma\left(\frac{n}{4}\right)+T(n), & \text { if } n \equiv 4(\bmod 8), \\
\frac{1}{8} \sigma_{5}\left(\frac{n}{4}\right)-\frac{33}{4} \sigma_{5}\left(\frac{n}{8}\right)+16 \sigma_{5}\left(\frac{n}{16}\right) & \\
\quad+\frac{3}{4} \sigma_{3}\left(\frac{n}{2}\right)-\frac{27}{2} \sigma_{3}\left(\frac{n}{4}\right)+24 \sigma_{3}\left(\frac{n}{8}\right)+\frac{3}{16} \bar{\sigma}(n), & \text { if } n \equiv 0(\bmod 8) .\end{cases}
\end{aligned}
$$


Table 1 Identities of convolution sums

\begin{tabular}{|c|c|}
\hline Identities of convolution sums & Reference \\
\hline$\overline{\sum_{k=1}^{n_{1}-1} \sigma_{1}(k) \sigma_{1}\left(n_{1}-k\right)}$ & (1) \\
\hline$\sum_{k=1}^{n_{1}-1} \bar{\sigma}(k) \bar{\sigma}\left(n_{1}-k\right)$ & Theorem 4 \\
\hline$\sum_{\substack{a_{1}+a_{2}+a_{3}=n_{2} \\
a_{1}, a_{2}, a_{3} \geq 1}} \sigma\left(a_{1}\right) \sigma\left(a_{2}\right) \sigma\left(a_{3}\right)$ & [11], [20, p. 148] \\
\hline$\sum_{\substack{a_{1}+a_{2}+a_{3}=n_{2} \\
a_{1}, a_{2}, a_{3} \geq 1}} \bar{\sigma}\left(a_{1}\right) \bar{\sigma}\left(a_{2}\right) \bar{\sigma}\left(a_{3}\right)$ & Theorem 5 \\
\hline$\sum_{\substack{1 \\
a_{1}+a_{2}+a_{3}+a_{4}=n_{3} \\
a_{1}, a_{2}, a_{3}, a_{4} \geq 1}} \sigma\left(a_{1}\right) \sigma\left(a_{2}\right) \sigma\left(a_{3}\right) \sigma\left(a_{4}\right)$ & {$[11],[20$, p. 158] } \\
\hline 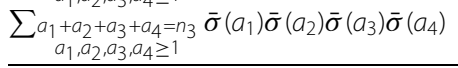 & Theorem 6 \\
\hline
\end{tabular}

Theorem 6 Let $n \geq 4$ be an integer. Then we have

$$
\begin{aligned}
& \sum_{\substack{a_{1}+a_{2}+a_{3}+a_{4}=n \\
a_{1}, a_{2}, a_{3}, a_{4} \geq 1}} \bar{\sigma}\left(a_{1}\right) \bar{\sigma}\left(a_{2}\right) \bar{\sigma}\left(a_{3}\right) \bar{\sigma}\left(a_{4}\right) \\
& = \begin{cases}(-1)^{\frac{n+1}{2}}\left(\frac{1}{256} \sigma_{5}(n)-\frac{1}{16} \sigma(n)\right)+T(n)-\frac{1}{16} \mathfrak{d}(n)-\frac{3}{16} \mathfrak{e}_{1}(n), & \text { if } n \text { is odd, } \\
-\frac{1}{136} \sigma_{7}\left(\frac{n}{2}\right)+\frac{3}{8} \sigma_{3}\left(\frac{n}{2}\right)+T(n)-\frac{1}{16} \mathfrak{d}(n)-\frac{2}{17} \mathfrak{e}_{2}(n), & \text { if } n \equiv 2(\bmod 4), \\
-\frac{1}{136} \sigma_{7}\left(\frac{n}{2}\right)+\frac{32}{17} \sigma_{7}\left(\frac{n}{4}\right)+\frac{1}{8} \sigma_{5}\left(\frac{n}{4}\right)+\frac{3}{8} \sigma_{3}\left(\frac{n}{2}\right)-\frac{27}{4} \sigma_{3}\left(\frac{n}{4}\right) & \\
+\frac{1}{8} \sigma\left(\frac{n}{4}\right)+T(n)-\frac{1}{16} \mathfrak{d}(n)-\frac{2}{17} \mathfrak{e}_{2}(n), & \\
-\frac{1}{136} \sigma_{7}\left(\frac{n}{2}\right)+\frac{32}{17} \sigma_{7}\left(\frac{n}{4}\right)+\frac{1}{8} \sigma_{5}\left(\frac{n}{4}\right)-\frac{33}{4} \sigma_{5}\left(\frac{n}{8}\right) & \text { if } n \equiv 4(\bmod 8), \\
+16 \sigma_{5}\left(\frac{n}{16}\right)+\frac{3}{8} \sigma_{3}\left(\frac{n}{2}\right)-\frac{27}{4} \sigma_{3}\left(\frac{n}{4}\right)+12 \sigma_{3}\left(\frac{n}{8}\right) & \text { if } n \equiv 0(\bmod 8) . \\
+\frac{1}{16} \bar{\sigma}(n)-\frac{1}{16} \mathfrak{d}(n)-\frac{2}{17} \mathfrak{e}_{2}(n), & \end{cases}
\end{aligned}
$$

Remark 2 Comparing old results on convolution sums with Theorem 4, 5, and 6, we have the Table 1.

For similar results, see [10], [16].

\section{Proof of Theorem 1}

In [4, p. 23], we find the curious identity

$$
\prod_{n \geq 1} \frac{\left(1-q^{n}\right)^{4}}{\left(1-2 q^{n} \cos u+q^{2 n}\right)^{2}}=1-8 \sin ^{2} \frac{u}{2} \sum_{N \geq 1} q^{N} \sum_{\substack{n k=N \\ n, k \geq 1}} n \cos (k-n) u \text {. }
$$

Putting $u=\frac{\pi}{2}$ into (13), we get

$$
\prod_{n \geq 1} \frac{\left(1-q^{n}\right)^{4}}{\left(1+q^{2 n}\right)^{2}}=1-4 \sum_{N \geq 1} q^{N} \sum_{\substack{n k=N \\ n, k \geq 1}} n \cos (k-n) \frac{\pi}{2} .
$$

We obtain three cases:

$$
n \cos (k-n) \frac{\pi}{2}= \begin{cases}n & \text { if }(k-n) \equiv 0(\bmod 4) \\ 0 & \text { if }(k-n) \equiv 1(\bmod 2) \\ -n & \text { if }(k-n) \equiv 2(\bmod 4)\end{cases}
$$


By (14) and (15) we get

$$
\prod_{n \geq 1} \frac{\left(1-q^{n}\right)^{4}}{\left(1+q^{2 n}\right)^{2}}=4 \sum_{k=1} q^{N}\left(\sum_{\substack{d k=N \\ d, k \geq 1 \\ k \equiv d(\bmod 4)}} d-\sum_{\substack{d k=N \\ d, k \geq 1 \\ k-d \equiv 2(\bmod 4)}} d\right)
$$

Therefore we get

$$
\prod_{n \geq 1} \frac{\left(1-q^{n}\right)^{4}}{\left(1+q^{2 n}\right)^{2}}=1-4 \sum_{N=1}^{\propto} \bar{\sigma}(N) q^{N}
$$

Lemma 1 Let $n \equiv 2(\bmod 4)$ be a positive integer. Then $\mathfrak{a}_{1}(n)=0$.

Proof Let $d$ be a positive divisor of $n$. If $d \equiv 1($ resp., 0$)(\bmod 2)$, then $\frac{n}{d} \equiv 0($ resp., 1$)$ $(\bmod 2)$. Thus we obtain $d-\frac{n}{d} \equiv 1(\bmod 2)$. We easily check that $\bar{\sigma}(n)=0$ and $\mathfrak{a}_{1}(n)=$ $-4 \bar{\sigma}(n)=0$. This completes the proof of Lemma 1 .

Lemma 2 Let $n \equiv 3(\bmod 4)$ be a positive integer. Then $\mathfrak{a}_{1}(n)=4 \sigma(n)>0$ and $\mathfrak{a}_{1}(n) \equiv$ $0(\bmod 16)$.

Proof If $d \equiv 1($ resp., 3$)(\bmod 4)$, then $\frac{n}{d} \equiv 3($ resp., 1$)(\bmod 4)$. Thus we obtain that $d-\frac{n}{d} \equiv$ $2(\bmod 4)$. Furthermore, there does not exist a pair $\left(d, \frac{n}{d}\right)$ satisfying $d \equiv \frac{n}{d}(\bmod 4)$. Hence

$$
\bar{\sigma}(n)=\sum_{\substack{d \mid n \\ d \equiv \frac{n}{d}(\bmod 4)}} d-\sum_{\substack{d \mid n \\ d-\frac{n}{d} \equiv 2(\bmod 4)}} d=-\sum_{\substack{d \mid n \\ d-\frac{n}{d} \equiv 2(\bmod 4)}} d=-\sum_{d \mid n} d=-\sigma(n)
$$

and $\mathfrak{a}_{1}(n)=-4 \bar{\sigma}(n)=4 \sigma(n)>0$. Note that

$$
\bar{\sigma}(n)=-\sum_{\substack{d \mid n \\ d-\frac{n}{d} \equiv 2(\bmod 4)}} d=-\sum_{\substack{d \mid n \\ d<\frac{n}{2} \\ d-\frac{n}{d} \equiv 2(\bmod 4)}} d-\sum_{\substack{d \mid n \\ d<\frac{n}{2} \\ d-\frac{n}{d} \equiv 2(\bmod 4)}} \frac{n}{d}=-\sum_{\substack{d \mid n \\ d<\frac{n}{2} \\ d-\frac{n}{d} \equiv 2(\bmod 4)}}\left(d+\frac{n}{d}\right)
$$

and

$$
d+\frac{n}{d} \equiv 0(\bmod 4)
$$

By (17) and (18) we get $\mathfrak{a}_{1}(n)=-4 \bar{\sigma}(n)=4 \sigma(n) \equiv 0(\bmod 16)$. This completes the proof of Lemma 2.

Lemma 3 Let $n \equiv 1(\bmod 4)$ be a positive integer. Then $\mathfrak{a}_{1}(n)=-4 \sigma(n)<0$. In particular,

$$
\mathfrak{a}_{1}(n) \equiv \begin{cases}4(\bmod 8) & \text { if } n \text { is a square integer } \\ 0(\bmod 8) & \text { otherwise. }\end{cases}
$$

Proof First, we consider the case where $n$ is a nonsquare integer. If $n \equiv 1(\bmod 4)$ is a nonsquare integer, then we write $n=p_{1}^{f_{1}} \cdots p_{r}^{f_{r}}$ for some $f_{i} \equiv 1(\bmod 2)$ with $1 \leq i \leq r$, 
where, $p_{i}$ are odd distinct prime integers. Since $d \equiv \frac{n}{d}(\bmod 4)$, we have

$$
\bar{\sigma}(n)=\sum_{\substack{d \mid n \\ d \equiv \frac{n}{d}(\bmod 4)}} d-\sum_{\substack{d \mid n \\ d-\frac{n}{d} \equiv 2(\bmod 4)}} d=\sum_{\substack{d \mid n \\ d \equiv \frac{n}{d}(\bmod 4)}} d=\sum_{d \mid n} d=\sigma(n) .
$$

On the other hand, we obtain $d \neq \frac{n}{d}$ when $d \mid n$. Since $d+\frac{n}{d} \equiv 0(\bmod 2)$, we note that

$$
\bar{\sigma}(n)=\sum_{\substack{d \mid n \\ d \equiv \frac{n}{d}(\bmod 4)}} d=\sum_{\substack{d \mid n \\ d<\frac{n}{2} \\ d \equiv \frac{n}{d}(\bmod 4)}}\left(d+\frac{n}{d}\right) \equiv 0(\bmod 2) .
$$

Therefore $\mathfrak{a}_{1}(n)=-4 \bar{\sigma}(n)=-4 \sigma(n)<0$ and $\mathfrak{a}_{1}(n) \equiv 0(\bmod 8)$. Second, we consider the case where $n$ is a square integer. Let $n=p_{1}^{2 e_{1}} \cdots p_{r}^{2 e_{r}}$. In this case, all factors of $n$ have their pairs $\left(d, \frac{n}{d}\right)$ satisfying $d \neq \frac{n}{d}$ except for $p_{1}^{f_{1}} \cdots p_{r}^{f_{r}}$. So,

$$
\bar{\sigma}(n)=\left(\sum_{\substack{d \mid n \\ d \equiv \frac{n}{d}(\bmod 4) \\ d \neq \frac{n}{d}}} d\right)+p_{1}^{f_{1}} \cdots p_{r}^{f_{r}}=\sum_{\substack{d \mid n \\ d \neq \frac{n}{d} \\ d<\frac{n}{2}}}\left(d+\frac{n}{d}\right)+p_{1}^{f_{1}} \cdots p_{r}^{f_{r}} \equiv 1(\bmod 2)
$$

Thus $\mathfrak{a}_{1}(n)=-4 \bar{\sigma}(n)=-4 \sigma(n)<0$ and $\mathfrak{a}_{1}(n) \equiv 4(\bmod 8)$. These complete the proof of Lemma 3.

Lemma 4 Let $n=4 m$ be a positive integer with $(2, m)=1$. Then $\mathfrak{a}_{1}(n)=-8 \sigma\left(\frac{n}{4}\right)<0$ and

$$
\mathfrak{a}_{1}(n) \equiv \begin{cases}8(\bmod 16) & \text { if } m \text { is square } \\ 0(\bmod 16) & \text { otherwise }\end{cases}
$$

Proof Let $n=4 p_{1}^{e_{1}} \cdots p_{r}^{e_{r}}$ be a positive integer with odd distinct primes $p_{i}$. All odd divisors $d$ of $n$ satisfy $d-\frac{n}{d} \equiv 1(\bmod 2)$, so we do not consider them. Hence we only consider the divisor $d$ of $n$ satisfying $d \equiv \frac{n}{d} \equiv 2(\bmod 4)$, that is, we can choose $d=2 S_{1}$ and $\frac{n}{d}=2 S_{2}$ with $4 S_{1} S_{2}=n$, where, $S_{1} \equiv S_{2} \equiv 1(\bmod 2)$. Thus $d \equiv \frac{n}{d}(\bmod 4)$. So,

$$
\bar{\sigma}(n)=\sum_{\substack{d \mid n \\ d \equiv \frac{n}{d}(\bmod 4)}} d-\sum_{\substack{d \mid n \\ d-\frac{n}{d} \equiv 2(\bmod 4)}} d=\sum_{\substack{d \mid n \\ d \equiv \frac{n}{d}(\bmod 4)}} d=2 \sum_{S_{1} \mid \frac{n}{4}} S_{1}=2 \sigma\left(\frac{n}{4}\right)
$$

If $\frac{n}{4}$ is a square integer, then $\sigma\left(\frac{n}{4}\right) \equiv 1(\bmod 2)$ by [20, p. 28]. So, by $(20), \mathfrak{a}_{1}(n)=-4 \bar{\sigma}(n)=$ $-8 \sigma\left(\frac{n}{4}\right)<0$ with $\left(8, \sigma\left(\frac{n}{4}\right)\right)=1$. Therefore $\mathfrak{a}_{1}(n) \equiv 8(\bmod 16)$.

On the other hand, if $\frac{n}{4}$ is not a square, then $S_{1} \neq \frac{n}{4 S_{1}}$ for all $S_{1} \mid \frac{n}{4}$. It is obvious that

$$
\sigma\left(\frac{n}{4}\right)=\sum_{S_{1} \mid \frac{n}{4}} S_{1}=\sum_{\substack{S_{1} \mid \frac{n}{4} \\ S_{1}<\frac{n}{8}}}\left(S_{1}+\frac{n}{4 S_{1}}\right) \equiv 0(\bmod 2) .
$$

We have $\mathfrak{a}_{1}(n)=-8 \sigma\left(\frac{n}{4}\right) \equiv 0(\bmod 16)$ by $(20)$ and $(21)$. These complete the proof of Lemma 4. 
Using (20) and (21), we obtain a more general congruence of the result in Lemma 4.

Corollary 1 If $n=4 p_{1}^{f_{1}} \cdots p_{r}^{f_{r}}$ is a nonsquare integer, then $\sigma\left(\frac{n}{4}\right) \equiv 0\left(\bmod \left(e_{1}+1\right) \cdots\left(e_{r}+1\right)\right)$ and $\mathfrak{a}_{1}(n) \equiv 0\left(\bmod 8\left(e_{1}+1\right) \cdots\left(e_{r}+1\right)\right)$. Here $p_{i}$ are distinct odd prime integers.

Lemma 5 Let $n=2^{a} p_{1}^{e_{1}} \cdots p_{r}^{e_{r}}$ be a positive integer with $a \geq 3$. Then $\mathfrak{a}_{1}(n)>0$. In particular,

$$
\mathfrak{a}_{1}(n) \equiv \begin{cases}24(\bmod 48) & \text { if } \frac{n}{2^{a}} \text { is square } \\ 0(\bmod 48) & \text { otherwise }\end{cases}
$$

Proof Let $n=2^{a} m$ be an integer with $(m, 2)=1$ and $a \geq 3$. If $d \not \equiv \frac{n}{d}(\bmod 2)$, then we do not consider these divisors $d$ of $n$. Putting $n=2^{k} \cdot 2^{a-k} m$ with $1 \leq k \leq a-1$, assume that $S_{1} \mid m$. Then we get

$$
2 S_{1}-2^{a-1} \frac{m}{S_{1}} \equiv 2^{a-1} S_{1}-2 \frac{m}{S_{1}} \equiv 2(\bmod 4)
$$

and

$$
2^{k_{1}} S_{1}-2^{a-k_{1}} \frac{m}{S_{1}} \equiv 0(\bmod 4) \quad \text { with } 2 \leq k_{1} \leq a-2
$$

By (22) and (23) we get

$$
\sum_{\substack{d \mid n \\ d-\frac{n}{d} \equiv 2(\bmod 4)}} d=-\left(2 \sum_{d \mid m} d+2^{a-1} \sum_{d \mid m} d\right)=-\left(2+2^{a-1}\right) \sigma(m)
$$

and

$$
\sum_{\substack{d \mid n \\ d \equiv \frac{n}{d}(\bmod 4)}} d=\sum_{k=2}^{a-2} 2^{k}\left(\sum_{d \mid m} d\right)=\sum_{k=2}^{a-2} 2^{k} \sigma(m) .
$$

From (24) and (25) we get

$$
\bar{\sigma}(n)=-\left(2+2^{a-1}\right) \sigma(m)+\sum_{k=2}^{a-2} 2^{k} \sigma(m)=-6 \sigma(m) .
$$

Here we easily check that $\sigma(m)=\sigma_{1,1}\left(\frac{n}{2 j} ; 2\right)$ with $0 \leq j \leq a$. Therefore we obtain that

$$
\mathfrak{a}_{1}(n)=24 \sigma(m)=24 \sigma_{1,1}(n)=24 \sigma\left(\frac{n}{2^{a}}\right)>0
$$

On the other hand, by [20, p. 28] we obtain

$$
\sigma\left(\frac{n}{2^{a}}\right) \equiv \begin{cases}0(\bmod 2) & \text { if } \frac{n}{2^{a}} \text { is square } \\ 1(\bmod 2) & \text { otherwise. }\end{cases}
$$


By (27) and (28) we obtain

$$
\mathfrak{a}_{1}(n) \equiv \begin{cases}24(\bmod 48) & \text { if } \frac{n}{2^{a}} \text { is square } \\ 0(\bmod 48) & \text { otherwise }\end{cases}
$$

This completes the proof of Lemma 5.

Proof of Theorem 1 Using Lemmas 1, 2, 3, 4, and 5, we can get the proof of Theorem 1 .

\section{The proof of Theorem 2 and Theorem 3}

Glaisher [3, p. 300] proved that

$$
\sigma(1) \sigma(2 n-1)+\sigma(3) \sigma(2 n-3)+\cdots+\sigma(2 n-1) \sigma(1)=\frac{1}{8}\left(\sigma_{3}(2 n)-\sigma_{3}(n)\right) .
$$

In [20, p. 192], a more general formula for natural numbers $n$ is given:

$$
\begin{aligned}
\sum_{\substack{m<n \\
m \text { odd }}} \sigma(m) \sigma(n-m)= & \frac{5}{24} \sigma_{3}(n)-\frac{7}{8} \sigma_{3}\left(\frac{n}{2}\right)+\frac{2}{3} \sigma_{3}\left(\frac{n}{4}\right)+\left(\frac{1}{24}-\frac{1}{4} n\right) \sigma(n) \\
& -\left(\frac{1}{8}-\frac{3}{4} n\right) \sigma\left(\frac{n}{2}\right)+\left(\frac{1}{12}-\frac{1}{2} n\right) \sigma\left(\frac{n}{4}\right) .
\end{aligned}
$$

To prove Lemma 7 and 8, we need a Glaisher's result in [5, p. 11] and [9]:

$$
\sum_{k=1}^{n-1} \sigma_{1,1}(k ; 2) \sigma_{1,1}(n-k ; 2)=\frac{1}{24}\left(11 \sigma_{3}(n)-\sigma_{3}(2 n)-2 \sigma_{1,1}(n ; 2)\right) .
$$

Lemma 6 Let $n \in \mathbb{N} \cup\{0\}$. Then we have $\mathfrak{a}_{2}(4 n+2)>0$. In particular, $\mathfrak{a}_{2}(4 n+2)=$ $16 \sigma_{3}(2 n+1)$.

Proof By (8) we note that

$$
\begin{aligned}
\mathfrak{a}_{2}(4 n+2) & =\sum_{m=0}^{4 n+2} \mathfrak{a}_{1}(m) \mathfrak{a}_{1}(4 n+2-m) \\
& =\sum_{m=0}^{2 n+1} \mathfrak{a}_{1}(2 m) \mathfrak{a}_{1}(4 n+2-2 m)+\sum_{m=1}^{2 n+1} \mathfrak{a}_{1}(2 m-1) \mathfrak{a}_{1}(4 n+2-(2 m-1)) .
\end{aligned}
$$

If $2 m \equiv 2($ resp., 0$)(\bmod 4)$, then $(4 n+2-2 m) \equiv 0($ resp., 2$)(\bmod 4)$. It is easy to check that $\mathfrak{a}_{1}(2 m)=0$ or $\mathfrak{a}_{1}(4 n+2-2 m)=0$ by (1). Then we have $\sum_{m=0}^{2 n+1} \mathfrak{a}_{1}(2 m) \mathfrak{a}_{1}(4 n+2-2 m)=0$. If $2 m-1 \equiv 1$ (resp., 3$)(\bmod 4)$, then $(4 n+3-2 m) \equiv 1($ resp., 3$)(\bmod 4)$.

By (17) and (19) we obtain

$$
\begin{aligned}
\mathfrak{a}_{1}(2 m-1) \mathfrak{a}_{1}(4 n+2-(2 m-1)) & =(4 \sigma(2 m-1)) \cdot(4 \sigma(4 n+2-(2 m-1))) \\
& =16 \sigma(2 m-1) \sigma(4 n+2-(2 m-1))
\end{aligned}
$$


By (30) and (33) we have

$$
\begin{aligned}
\sum_{m=1}^{2 n+1} \mathfrak{a}_{1}(2 m-1) \mathfrak{a}_{1}(4 n+2-(2 m-1)) & =16 \sum_{m=1}^{2 n+1} \sigma(2 m-1) \sigma(4 n+2-(2 m-1)) \\
& =2\left(\sigma_{3}(4 n+2)-\sigma_{3}(2 n+1)\right)=16 \sigma_{3}(2 n+1) .
\end{aligned}
$$

This completes the proof of Lemma 6.

Lemma 7 Let $n \in \mathbb{N}$. Then we have $\mathfrak{a}_{2}(4 n)<0$ with $(n, 2)=1$. In particular, $\mathfrak{a}_{2}(4 n)=$ $-144 \sigma_{3}(n)$.

Proof By (8) we note that

$$
\begin{aligned}
\mathfrak{a}_{2}(4 n)= & \sum_{m=0}^{4 n} \mathfrak{a}_{1}(m) \mathfrak{a}_{1}(4 n-m) \\
= & 2 \mathfrak{a}_{1}(0) \mathfrak{a}_{1}(4 n)+\sum_{m=1}^{2 n} \mathfrak{a}_{1}(2 m-1) \mathfrak{a}_{1}(4 n-(2 m-1)) \\
& +\sum_{m=1}^{n} \mathfrak{a}_{1}(4 m-2) \mathfrak{a}_{1}(4 n-4 m+2)+\sum_{m=1}^{n-1} \mathfrak{a}_{1}(4 m) \mathfrak{a}_{1}(4 n-4 m) .
\end{aligned}
$$

First, from Lemma 4 we find

$$
2 \mathfrak{a}_{1}(0) \mathfrak{a}_{1}(4 n)=2 \cdot 1 \cdot \mathfrak{a}_{1}(4 n)=-16 \sigma(n) .
$$

Second, we can consider $\sum_{\text {odd }} \mathfrak{a}_{1}(2 m-1) \mathfrak{a}_{1}(4 n-(2 m-1))$.

If $2 m-1 \equiv 1$ (resp., 3$)(\bmod 4)$, then $4 n-(2 m-1) \equiv 3(\operatorname{resp} ., 1)(\bmod 4)$. So, $\mathfrak{a}_{1}(2 m-$ 1) $\mathfrak{a}_{1}(4 n-(2 m-1))=-16 \sigma(2 m-1) \sigma(4 n-(2 m-1))$. Thus we note that

$$
\begin{aligned}
\sum_{m=1}^{2 n} \mathfrak{a}_{1}(2 m-1) \mathfrak{a}_{1}(4 n-(2 m-1)) & =-16 \sum_{m=1}^{2 n} \sigma(2 m-1) \sigma(4 n-(2 m-1)) \\
& =-2\left(\sigma_{3}(4 n)-\sigma_{3}(2 n)\right)=-128 \sigma_{3}(n)
\end{aligned}
$$

by (30).

Third, by Lemma 1 we obtain

$$
\sum_{m=1}^{n} \mathfrak{a}_{1}(4 m-2) \mathfrak{a}_{1}(4 n-4 m+2)=0
$$

Finally, by (16) we have to check that

$$
\sum_{m=1}^{n-1} \mathfrak{a}_{1}(4 m) \mathfrak{a}_{1}(4 n-4 m)=16 \sum_{m=1}^{n-1} \bar{\sigma}(4 m) \bar{\sigma}(4 n-4 m)
$$


If $m \equiv 1($ resp., 0$)(\bmod 2)$, then $4 m \equiv 4($ resp., 0$)(\bmod 8)$ and $4 n-4 m \equiv 0($ resp., 4$)$ $(\bmod 8)$. Thus by $(20)$ and $(26)$ we obtain

$$
\bar{\sigma}(4 m) \bar{\sigma}(4 n-4 m)=-12 \sigma_{1,1}(m ; 2) \sigma_{1,1}(n-m ; 2) .
$$

By (32), (38), and (39) we obtain

$$
\begin{aligned}
\sum_{m=1}^{n-1} \mathfrak{a}_{1}(4 m) \mathfrak{a}_{1}(4 n-4 m) \\
\quad=-12 \cdot\left(16 \sum_{m=1}^{n-1} \sigma_{1,1}(m ; 2) \sigma_{1,1}(n-m ; 2)\right) \\
\quad=-8\left(11 \sigma_{3}(n)-\sigma_{3}(2 n)-2 \sigma_{1,1}(n ; 2)\right)=-16\left(\sigma_{3}(n)-\sigma(n)\right) .
\end{aligned}
$$

It is well known that $\sigma_{1,1}(n ; 2)=\sigma_{1}(n)$ for odd $n$. Therefore the proof of Lemma 7 is completed by (34), (35), (36), (37), and (40).

To simplify Lemma 8, we introduce a useful formula from [20, p. 26]. Let $p$ be a prime. For $k, n \in \mathbb{N}$, we have

$$
\sigma_{k}(p n)-\left(p^{k}+1\right) \sigma_{k}(n)+p^{k} \sigma_{k}\left(\frac{n}{p}\right)=0
$$

Lemma 8 Let $n \in \mathbb{N}$. Then we have $\mathfrak{a}_{2}(8 n)=-16\left(\sigma_{3}(2 n)-25 \sigma_{3}(n)\right)>0$.

Proof By (8) we note that

$$
\begin{aligned}
\mathfrak{a}_{2}(8 n)= & \sum_{m=0}^{8 n} \mathfrak{a}_{1}(m) \mathfrak{a}_{1}(8 n-m) \\
= & 2 \mathfrak{a}_{1}(0) \mathfrak{a}_{1}(8 n)+\sum_{m=1}^{4 n} \mathfrak{a}_{1}(2 m-1) \mathfrak{a}_{1}(8 n-(2 m-1)) \\
& +\sum_{m=1}^{2 n} \mathfrak{a}_{1}(4 m-2) \mathfrak{a}_{1}(8 n-4 m+2)+\sum_{m=1}^{n} \mathfrak{a}_{1}(8 m-4) \mathfrak{a}_{1}(8 n-8 m+4) \\
& +\sum_{m=1}^{n-1} \mathfrak{a}_{1}(8 m) \mathfrak{a}_{1}(8 n-8 m) .
\end{aligned}
$$

First, by (27) we find

$$
2 \mathfrak{a}_{1}(0) \mathfrak{a}_{1}(8 n)=2 \cdot 1 \cdot \mathfrak{a}_{1}(8 n)=48 \sigma_{1,1}(n ; 2) .
$$

Second, using a similar method as for (36), we obtain

$$
\sum_{m=1}^{4 n} \mathfrak{a}_{1}(2 m-1) \mathfrak{a}_{1}(8 n-(2 m-1))=-2\left(\sigma_{3}(8 n)-\sigma_{3}(4 n)\right) .
$$


Third, by Lemma 1 we obtain

$$
\sum_{m=1}^{2 n} \mathfrak{a}_{1}(4 m-2) \mathfrak{a}_{1}(8 n-4 m+2)=0
$$

Note that $8 m-4 \equiv 8 n-8 m+4 \equiv 4(\bmod 8)$ and $\mathfrak{a}_{1}(8 m-4) \mathfrak{a}_{1}(8 n-8 m+4)=64 \sigma(2 m-$ 1) $\sigma(2 n-2 m+1)$ by Lemma 4 .

Forth, by (30) we get

$$
\begin{aligned}
\sum_{m=1}^{n} \mathfrak{a}_{1}(8 m-4) \mathfrak{a}_{1}(8 n-8 m+4) & =64 \sum_{m=1}^{n} \sigma(2 m-1) \sigma(2 n-(2 m-1)) \\
& =8\left(\sigma_{3}(2 n)-\sigma_{3}(n)\right) .
\end{aligned}
$$

Fifth, by (27) and (32) we have

$$
\begin{aligned}
\sum_{m=1}^{n-1} \mathfrak{a}_{1}(8 m) \mathfrak{a}_{1}(8 n-8 m) & =(24)^{2} \sum_{m=1}^{n-1} \sigma_{1,1}(m ; 2) \sigma_{1,1}(n-m ; 2) \\
& =24\left(11 \sigma_{3}(n)-\sigma_{3}(2 n)-2 \sigma_{1,1}(n ; 2)\right)
\end{aligned}
$$

It is well known that

$$
\sigma_{3}(8 n)=9 \sigma_{3}(4 n)-8 \sigma_{3}(2 n) \text { and } \sigma_{3}(4 n)=9 \sigma_{3}(2 n)-8 \sigma_{3}(n)
$$

by (41). By (42)-(47) we obtain

$$
\mathfrak{a}_{2}(8 n)=-16\left(\sigma_{3}(2 n)-25 \sigma_{3}(n)\right) .
$$

Let $n=2^{t} u$ be an integer with $(u, 2)=1$. Then

$$
\sigma_{3}(2 n)-25 \sigma_{3}(n)= \begin{cases}-16 \sigma_{3}(n) & \text { if } t=0 \\ \frac{1}{7}\left(-17 \cdot 2^{3 t+3}+24\right) \sigma_{3}(u) & \text { if } t \geq 1\end{cases}
$$

If $t \geq 1$ (resp., $=0$ ), then $\sigma_{3}(2 n)-25 \sigma_{3}(n) \leq-152 \sigma_{3}(u)$ (resp., $-16 \sigma_{3}(n)$ ). Therefore $\mathfrak{a}_{2}(8 n)>0$. This completes the proof of Lemma 8 .

Corollary 2 If $n$ is an odd integer, then $\mathfrak{a}_{2}(8 n)=256 \sigma_{3}(n)$.

Proof of Theorem 2 We can get the proof of the theorem by using Lemmas 6-8 and Corollary 2,

Corollary 3 Let $n, M, N \in \mathbb{N}$ with $N \equiv 1(\bmod 2)$ and $M \geq 3$.

(1) $\sum_{m=1}^{4 n+1} m \mathfrak{a}_{1}(m) \mathfrak{a}_{1}(4 n+2-m)=(2 n+1) \sigma_{3}(2 n+1)$.

(2) $\sum_{m=1}^{8 n-1} m \mathfrak{a}_{1}(m) \mathfrak{a}_{1}(8 n-m)=-64 n\left(\sigma_{3}(2 n)-25 \sigma_{3}(n)+6 \sigma(n)-12 \sigma\left(\frac{n}{2}\right)\right)$.

(3) $\sum_{m=1}^{4 N-1} m \mathfrak{a}_{1}(m) \mathfrak{a}_{1}(4 N-m)=-32 N\left(9 \sigma_{3}(N)-\sigma(N)\right)$.

(4) $\sum_{k_{1}+k_{2}+k_{3}=M} \mathfrak{a}_{1}\left(8 k_{1}\right) \mathfrak{a}_{1}\left(8 k_{2}\right) \mathfrak{a}_{1}\left(8 k_{3}\right)=$ $-18\left(29 \sigma_{5}(M)-\sigma_{5}(2 M)+44 \sigma_{3}(M)-4 \sigma_{3}(2 M)-4 \sigma(M)+8 \sigma\left(\frac{M}{2}\right)\right)$.

(5) $\sum_{k_{1}+\cdots k_{l}=N} \mathfrak{a}_{1}\left(2 k_{1}\right) \cdots \mathfrak{a}_{1}\left(2 k_{l}\right)=0$. 
Proof (1) We easily check that

$$
\sum_{m=1}^{4 n+1} m \mathfrak{a}_{1}(m) \mathfrak{a}_{1}(4 n+2-m)=\sum_{m=1}^{4 n+1}(4 n+2-m) \mathfrak{a}_{1}(m) \mathfrak{a}_{1}(4 n+2-m) .
$$

Thus it is clear by Lemma 6.

(2) This is easily proved by using the same method as for (49) and then Lemma 8 and $(42)$.

(3) It is obtained by using the same method as for (49) and then Lemma 7 and (35).

(4) By (27) we obtain

$$
\sum_{k_{1}+k_{2}+k_{3}=M} \mathfrak{a}_{1}\left(8 k_{1}\right) \mathfrak{a}_{1}\left(8 k_{2}\right) \mathfrak{a}_{1}\left(8 k_{3}\right)=(24)^{3} \sum_{k_{1}+k_{2}+k_{3}=M} \sigma_{1,1}\left(k_{1} ; 2\right) \sigma_{1,1}\left(k_{2} ; 2\right) \sigma_{1,1}\left(k_{3} ; 2\right) .
$$

It is obtained by [9, Theorem 3.7].

(5) If $k_{i} \equiv 0(\bmod 2)$ for all $1 \leq i \leq l$, then $N \equiv 0(\bmod 2)$. This contradicts the fact that $N$ is an odd integer. Thus at least one odd integer $k_{i}$ exists, which is clear by Lemma 1.

To prove Theorem 3, we need the following lemma.

Lemma 9 Let $n \in \mathbb{N}$. Then we have $\mathfrak{a}_{2}(2 n-1)=-8 \mathfrak{e}_{1}(2 n-1)$.

Proof Let

$$
\begin{aligned}
f(q) & :=\left(\prod_{n \geq 1} \frac{\left(1-q^{n}\right)^{4}}{\left(1+q^{2 n}\right)^{2}}\right)^{2}+8 q \prod_{n \geq 1}\left(1-q^{2 n}\right)^{4}\left(1-q^{4 n}\right)^{4} \\
& =\sum_{n \text { even }} u(n) q^{n}+\sum_{n \text { odd }} v(n) q^{n}
\end{aligned}
$$

To prove Lemma 9, we have to prove that $f(q)$ is an even function, that is, $f(q)-f(-q)=0$. By the Jacobi product identity [6, Theorem 3.9] we obtain that

$$
\begin{aligned}
f(q) & -f(-q) \\
:= & \prod_{n \geq 1} \frac{\left(1-q^{2 n}\right)^{8}}{\left(1+q^{2 n}\right)^{4}}\left(\prod_{n \geq 1}\left(1-q^{2 n-1}\right)^{8}-\prod_{n \geq 1}\left(1+q^{2 n-1}\right)^{8}\right)+16 q \prod_{n \geq 1}\left(1-q^{2 n}\right)^{4}\left(1-q^{4 n}\right)^{4} \\
= & \prod_{n \geq 1} \frac{\left(1-q^{2 n}\right)^{8}}{\left(1+q^{2 n}\right)^{4}}\left(-16 q \prod_{n \geq 1}\left(1+q^{2 n}\right)^{8}\right)+16 q \prod_{n \geq 1}\left(1-q^{2 n}\right)^{4}\left(1-q^{4 n}\right)^{4}=0 .
\end{aligned}
$$

Thus we obtain that $\mathfrak{a}_{2}(2 n-1)=v(2 n-1)=-8 \mathfrak{e}_{1}(2 n-1)$.

Proof of Theorem 3 Since

$$
\sum_{m \geq 0} \mathfrak{e}_{r}(m) q^{m}=\left(\sum_{m \geq 0} \mathfrak{e}_{1}(m) q^{m}\right)^{r}=\left(\sum_{\substack{m \geq 0 \\ m \text { odd }}} \mathfrak{e}_{1}(m) q^{m}\right)^{r}
$$


we have

$$
\mathfrak{e}_{r}(m)=\sum_{\substack{a_{1}+a_{2}+\cdots+a_{r}=m \\ a_{1}, a_{2}, \ldots, a_{r} \text { odd }}} \mathfrak{e}_{1}\left(a_{1}\right) \mathfrak{e}_{1}\left(a_{2}\right) \cdots \mathfrak{e}_{1}\left(a_{r}\right)
$$

From Lemma 9 we have $\mathfrak{a}_{2}(m)=-8 \mathfrak{e}_{1}(m)$ for $m$ odd. Thus we get

$$
\begin{aligned}
\mathfrak{e}_{r}(m) & =\left(-\frac{1}{8}\right)^{r} \sum_{\substack{a_{1}+a_{2}+\cdots+a_{r}=m \\
a_{1}, a_{2}, \ldots, a_{r} \text { odd }}} \mathfrak{a}_{2}\left(a_{1}\right) \mathfrak{a}_{2}\left(a_{2}\right) \cdots \mathfrak{a}_{2}\left(a_{r}\right) \\
& =\left(-\frac{1}{8}\right)^{r} \sum_{\substack{a_{1}+a_{2}+\cdots+a_{r}=m \\
a_{1}, a_{2}, \ldots, a_{r} \text { odd }}}\left(\sum_{t_{1}=0}^{a_{1}} \mathfrak{a}_{1}\left(t_{1}\right) \mathfrak{a}_{1}\left(a_{1}-t_{1}\right) \cdots \sum_{t_{r}=0}^{a_{r}} \mathfrak{a}_{1}\left(t_{r}\right) \mathfrak{a}_{1}\left(a_{r}-t_{r}\right)\right) .
\end{aligned}
$$

Recall that $\mathfrak{a}_{1}(0)=1$ and $\mathfrak{a}_{1}(n)=-4 \bar{\sigma}(n)$ for $n \geq 1$ from (16). Then we have

$$
\sum_{\substack{a_{1}+a_{2}+\cdots+a_{r}=m \\ a_{1}, a_{2}, \ldots, a_{r} \text { odd } \\ 0 \leq t_{i} \leq a_{i}}}\left(-\frac{1}{4}\right)^{\epsilon\left(t_{1}, a_{1}-t_{1}, \ldots, t_{r}, a_{r}-t_{r}\right)} \bar{\sigma}\left(t_{1}\right) \bar{\sigma}\left(a_{1}-t_{1}\right) \cdots \bar{\sigma}\left(t_{r}\right) \bar{\sigma}\left(a_{r}-t_{r}\right)=\left(-\frac{1}{2}\right)^{r} \mathfrak{c}_{r}(m)
$$

where $\epsilon\left(t_{1}, a_{1}-t_{1}, \ldots, t_{r}, a_{r}-t_{r}\right):=\#\left\{t_{i}=0\right.$ or $\left.a_{j}-t_{j}=0 \mid 1 \leq i, j \leq r\right\}$. Also, we can obtain another expression:

$$
\begin{aligned}
\mathfrak{e}_{r}(m) & =\left(-\frac{1}{8}\right)^{r} \sum_{\substack{a_{1}+a_{2}+\cdots+a_{r}=m \\
a_{1}, a_{2}, \ldots, a_{r} \text { odd }}} \prod_{i=1}^{r}\left(-8 \bar{\sigma}\left(a_{i}\right)+16 \sum_{\substack{t_{i}=1 \\
a_{i} \neq 1}}^{a_{i}-1} \bar{\sigma}\left(t_{i}\right) \bar{\sigma}\left(a_{i}-t_{i}\right)\right) \\
& =\sum_{\substack{a_{1}+a_{2}+\cdots+a_{r}=m \\
a_{1}, a_{2}, \ldots, a_{r} \text { odd }}} \prod_{i=1}^{r}\left(\bar{\sigma}\left(a_{i}\right)-2 \sum_{\substack{t_{i}=1 \\
a_{i} \neq 1}}^{a_{i}-1} \bar{\sigma}\left(t_{i}\right) \bar{\sigma}\left(a_{i}-t_{i}\right)\right) .
\end{aligned}
$$

\section{The proof of Theorem 4}

Proof of Theorem 4 We will use the theory of modular forms. In fact, $F(q)^{2}=$ $\sum_{m \geq 0} \mathfrak{a}_{2}(m) q^{m}$ is in the space $M_{4}\left(\Gamma_{0}(8)\right)$, which is a five-dimensional vector space; see [8, Theorem 3.8]. Let

$$
E_{4}(q)=1+240 \sum_{n=1}^{\infty} \sigma_{3}(n) q^{n}
$$

Then the space $M_{4}\left(\Gamma_{0}(8)\right)$ is spanned by $E_{4}(q), E_{4}\left(q^{2}\right), E_{4}\left(q^{4}\right), E_{4}\left(q^{8}\right)$, and $Y(q)$. Comparing the Fourier coefficients, we have

$$
\begin{aligned}
F(q)^{2} & =\frac{1}{15} E_{4}\left(q^{2}\right)-\frac{6}{5} E_{4}\left(q^{4}\right)+\frac{32}{15} E_{4}\left(q^{8}\right)-8 Y(q) \\
& =1+\sum_{n=1}^{\infty}\left(16 \sigma_{3}\left(\frac{n}{2}\right)-288 \sigma_{3}\left(\frac{n}{4}\right)+512 \sigma_{3}\left(\frac{n}{8}\right)-8 \mathfrak{e}_{1}(n)\right) q^{n},
\end{aligned}
$$

and then $\mathfrak{a}_{2}(n)=16 \sigma_{3}\left(\frac{n}{2}\right)-288 \sigma_{3}\left(\frac{n}{4}\right)+512 \sigma_{3}\left(\frac{n}{8}\right)-8 \mathfrak{e}_{1}(n)$ for $n \geq 1$. In particular, $\mathfrak{a}_{2}(n)=$ $-8 \mathfrak{e}_{1}(n)$ for $n$ odd. The same result is in Lemma 9. 
Meanwhile, since

$$
\begin{aligned}
F(q)^{2} & =\sum_{n \geq 0} \sum_{k=0}^{n} \mathfrak{a}_{1}(k) \mathfrak{a}_{1}(n-k) q^{n}=1+\sum_{n \geq 1}\left(2 \mathfrak{a}_{1}(n)+\sum_{\substack{k=1 \\
n \neq 1}}^{n-1} \mathfrak{a}_{1}(k) \mathfrak{a}_{1}(n-k)\right) q^{n} \\
& =1+\sum_{n \geq 1}\left(-8 \bar{\sigma}(n)+16 \sum_{\substack{k=1 \\
n \neq 1}}^{n-1} \bar{\sigma}(k) \bar{\sigma}(n-k)\right) q^{n}
\end{aligned}
$$

for $n \geq 2$, we have

$$
\sum_{k=1}^{n-1} \bar{\sigma}(k) \bar{\sigma}(n-k)=\sigma_{3}\left(\frac{n}{2}\right)-18 \sigma_{3}\left(\frac{n}{4}\right)+32 \sigma_{3}\left(\frac{n}{8}\right)+\frac{1}{2} \bar{\sigma}(n)-\frac{1}{2} \mathfrak{e}_{1}(n) .
$$

\section{Several convolution sums}

To prove Lemmas 10, 11, and 13, we need the following propositions.

Proposition 1 ([9, Lemma 4.1, Corollary 4.7]) Let $N(\geq 3)$ be an integer.

(1) $\sum_{m=1}^{N} \sigma_{3}(2 m-1) \sigma(2 N-2 m+1)=\frac{1}{32}\left(\sigma_{5}(2 N)-\sigma_{5}(N)\right)$.

(2) $\sum_{m=1}^{N-1} \sigma_{3}(m) \sigma_{1,1}(N-m ; 2)=\frac{1}{240}\left(11 \sigma_{5}(N)-32 \sigma_{5}\left(\frac{N}{2}\right)-10 \sigma_{3}(N)-\sigma(N)+2 \sigma\left(\frac{N}{2}\right)\right)$.

(3) $\sum_{m=1}^{m<\frac{N}{2}} \sigma_{3}(m) \sigma_{3}(N-2 m)=\frac{1}{2040} \sigma_{7}(N)+\frac{2}{255} \sigma_{7}\left(\frac{N}{2}\right)-\frac{1}{240} \sigma_{3}(N)-\frac{1}{240} \sigma_{3}\left(\frac{N}{2}\right)+\frac{1}{272} \mathfrak{d}(N)$.

(4) $\sum_{m=1}^{m<\frac{N}{4}} \sigma_{3}(m) \sigma_{3}(N-4 m)=$ $\frac{1}{32,640} \sigma_{7}(N)+\frac{1}{2176} \sigma_{7}\left(\frac{N}{2}\right)+\frac{2}{255} \sigma_{7}\left(\frac{N}{4}\right)-\frac{1}{240} \sigma_{3}(N)-\frac{1}{240} \sigma_{3}\left(\frac{N}{4}\right)+\frac{9}{2176} \mathfrak{d}(N)+\frac{9}{136} \mathfrak{d}\left(\frac{N}{2}\right)$.

Proposition 2 Let $N(\geq 3)$ be an odd integer.

(1) $\sum_{m=1}^{N} \sigma_{3}(2 m-1) \sigma(2 N-2 m+1)=\sigma_{5}(N)$.

(2) $\sum_{m=1}^{N-1}\left(\sigma_{3}(2 m)-\sigma_{3}(m)\right) \sigma_{1,1}(N-m ; 2)=\frac{1}{3}\left(\sigma_{5}(N)-\sigma_{3}(N)\right)$.

(3) $\sum_{m=1}^{N-1} \sigma_{3}(m) \sigma_{1,1}(N ; 2)=\frac{1}{240}\left(11 \sigma_{5}(N)-10 \sigma_{3}(N)-\sigma(N)\right)$.

(4) $\sum_{m<\frac{N}{2}} \sigma_{3}(2 m) \sigma(N-2 m)=\frac{17}{480} \sigma_{5}(N)-\frac{1}{240} \sigma(N)-\frac{1}{32} \mathfrak{c}(N)$.

Proof (1)-(3) See [7, Corollary 3] and [9, Proposition 3.11, 3.12].

(4) In 1997, Melfi [13], [14] proved that

$$
\sum_{m<\frac{N}{2}} \sigma_{3}(m) \sigma(N-2 m)=\frac{1}{240}\left(\sigma_{5}(N)-\sigma(N)\right)
$$

In 2005, Cheng and Williams [2, Theorem 4.2 (iii)] showed that

$$
\sum_{m<\frac{N}{4}} \sigma_{3}(m) \sigma(N-4 m)=\frac{1}{3840} \sigma_{5}(N)-\frac{1}{240} \sigma(N)+\frac{1}{256} \mathfrak{c}(N) .
$$

By (41) we have

$$
\sum_{m=1}^{N-1} \sigma_{3}(2 m) \sigma(N-2 m)=9 \sum_{m=1}^{N-1} \sigma_{3}(m) \sigma(N-2 m)-8 \sum_{m=1}^{N-1} \sigma_{3}\left(\frac{m}{2}\right) \sigma(N-2 m) .
$$

From (53), (54), and (55) we obtain the desired result. 
Lemma 10 If $N$ is an integer, then $\sum_{m=1}^{4 N-1} \mathfrak{a}_{2}(2 m) \mathfrak{a}_{1}(8 N-2 m)=\frac{16}{5}\left(22 \sigma_{5}(2 N)-274 \sigma_{5}(N)+\right.$ $\left.5 \sigma_{3}(2 N)-125 \sigma_{3}(N)-12 \sigma(2 N)+24 \sigma(N)\right)$.

In particular, if $N$ is an odd integer, then $\sum_{m=1}^{4 N-1} \mathfrak{a}_{2}(2 m) \mathfrak{a}_{1}(8 N-2 m)=\frac{64}{5}\left(113 \sigma_{5}(N)-\right.$ $\left.25 \sigma_{3}(n)-3 \sigma(n)\right)$.

Proof By Lemma 1, (41), and Proposition 1 we obtain

$$
\begin{aligned}
& \sum_{m=1}^{4 N-1} \mathfrak{a}_{2}(2 m) \mathfrak{a}_{1}(8 N-2 m) \\
& \quad=\sum_{m=1}^{N} \mathfrak{a}_{2}(8 m-4) \mathfrak{a}_{1}(8 N-8 m+4)+\sum_{m=1}^{N-1} \mathfrak{a}_{2}(8 m) \mathfrak{a}_{1}(8 N-8 m) \\
& \quad=1152 \sum_{m=1}^{N} \sigma_{3}(2 m-1) \sigma(2 N-2 m+1)-384 \sum_{m=1}^{N-1}\left(\sigma_{3}(2 m)-25 \sigma_{3}(m)\right) \sigma_{1,1}(N-m ; 2) \\
& \quad=\frac{16}{5}\left(22 \sigma_{5}(2 N)-274 \sigma_{5}(N)+5 \sigma_{3}(2 N)-125 \sigma_{3}(N)-12 \sigma(2 N)+24 \sigma(N)\right)
\end{aligned}
$$

Similarly, by Lemma 1 and Proposition 2, for odd integers $N$, we obtain

$$
\begin{aligned}
& \sum_{m=1}^{4 N-1} \mathfrak{a}_{2}(2 m) \mathfrak{a}_{1}(8 N-2 m) \\
& =1152 \sigma_{5}(N)-384 \sum_{m=1}^{N-1}\left(\sigma_{3}(2 m)-\sigma_{3}(m)\right) \sigma_{1,1}(N-m ; 2) \\
& \quad+9216 \sum_{m=1}^{N-1} \sigma_{3}(m) \sigma_{1,1}(N-m ; 2) \\
& =\frac{64}{5}\left(113 \sigma_{5}(N)-20 \sigma_{3}(N)-3 \sigma(N)\right) .
\end{aligned}
$$

In fact, even if Lemma 1 and Proposition 2 are not used, this equation is easily induced by $\sigma_{5}(2)=33, \sigma_{3}(2)=9$, and $\sigma(2)=3$.

Lemma 11 If $N$ is an integer, then $\sum_{m=1}^{4 N+1} \mathfrak{a}_{2}(2 m) \mathfrak{a}_{1}(8 N+4-2 m)=-16\left(2 \sigma_{5}(2 N+1)-\right.$ $\left.9 \sigma_{3}(2 N+1)+7 \mathfrak{c}(2 N+1)\right)$.

Proof Let

$$
\begin{aligned}
& T_{1}:=-3456 \sum_{m=1}^{N} \sigma_{3}(2 m-1) \sigma(2 N+1-(2 m-1)), \\
& T_{3}:=128 \sum_{m=1}^{N} \sigma_{3}(2 m) \sigma(2 N+1-2 m), \\
& T_{2}:=6912 \sum_{m=1}^{N} \sigma_{3}(2 m-1) \sigma\left(\frac{2 N+1-(2 m-1)}{2}\right), \\
& T_{4}:=-128 \sum_{m=1}^{N} \sigma_{3}(m) \sigma(2 N+1-2 m) .
\end{aligned}
$$


By Lemma 1, Lemma 4, and (27) we have

$$
\begin{aligned}
& \sum_{m=1}^{4 N+1} \mathfrak{a}_{2}(2 m) \mathfrak{a}_{1}(8 N+4-2 m) \\
& \quad=\sum_{m=1}^{N} \mathfrak{a}_{2}(8 m-4) \mathfrak{a}_{1}(8 N+4-(8 m-4))+\sum_{m=1}^{N} \mathfrak{a}_{2}(8 m) \mathfrak{a}_{1}(8 N+4-8 m) \\
& \quad=\sum_{i=1}^{4} T_{i} .
\end{aligned}
$$

Comparing $[2,(1.7)]$ with Proposition 2 (4), we obtain the formula

$$
\begin{aligned}
T_{1} & =-3456\left(\sum_{m=1}^{2 N} \sigma_{3}(m) \sigma(2 N+1-m)-\sum_{m=1}^{N} \sigma_{3}(2 m) \sigma(2 N+1-2 m)\right) \\
& =-36\left(5 \sigma_{5}(2 N+1)-8(1+3 N) \sigma_{3}(2 N+1)+3 \mathfrak{c}(2 N+1)\right) .
\end{aligned}
$$

From [9, Proposition 4.5] we see that integer $N$ satisfy

$$
\begin{aligned}
T_{2} & =6912 \sum_{m=1}^{2 N} \sigma_{3}(m) \sigma\left(\frac{2 N+1-m}{2}\right) \\
& =144\left(\sigma_{5}(2 N+1)-(1+6 N) \sigma_{3}(2 N+1)\right) .
\end{aligned}
$$

By Proposition 2 (4) we have

$$
T_{3}=\frac{4}{15}\left(17 \sigma_{5}(2 N+1)-2 \sigma(2 N+1)-15 \mathfrak{c}(2 N+1)\right)
$$

Using $\left[2, T_{3,1}(n)\right]$, we can rewrite $T_{4}$ as

$$
T_{4}=-\frac{8}{15}\left(17 \sigma_{5}(2 N+1)-\sigma(2 N+1)\right)
$$

Finally, we apply (56)-(59) to get the result.

Now we change our direction to modular forms to see $T(n)$ defined in (12).

Lemma 12 If $n \equiv 0(\bmod 8)$, then $T(n)=0$.

Proof The space of cusp forms $S_{6}\left(\Gamma_{0}(16)\right)$ is a seven-dimensional vector space. Explicitly, this space is spanned by the following seven eta quotients:

$$
\begin{aligned}
& \frac{\eta(z)^{12} \eta(4 z)^{6}}{\eta(2 z)^{6}}=q \prod_{n=1}^{\infty} \frac{\left(1-q^{n}\right)^{12}\left(1-q^{4 n}\right)^{6}}{\left(1-q^{2 n}\right)^{6}}=\sum_{n=1}^{\infty} t_{1}(n) q^{n}, \\
& \frac{\eta(z)^{10} \eta(4 z)^{6} \eta(16 z)^{2}}{\eta(2 z)^{5} \eta(8 z)}=q^{2} \prod_{n=1}^{\infty} \frac{\left(1-q^{n}\right)^{10}\left(1-q^{4 n}\right)^{6}\left(1-q^{16 n}\right)^{2}}{\left(1-q^{2 n}\right)^{5}\left(1-q^{8 n}\right)}=\sum_{n=1}^{\infty} t_{2}(n) q^{n}, \\
& \frac{\eta(z)^{8} \eta(4 z)^{6} \eta(16 z)^{4}}{\eta(2 z)^{4} \eta(8 z)^{2}}=q^{3} \prod_{n=1}^{\infty} \frac{\left(1-q^{n}\right)^{8}\left(1-q^{4 n}\right)^{6}\left(1-q^{16 n}\right)^{4}}{\left(1-q^{2 n}\right)^{4}\left(1-q^{8 n}\right)^{2}}=\sum_{n=1}^{\infty} t_{3}(n) q^{n},
\end{aligned}
$$




$$
\begin{aligned}
& \frac{\eta(z)^{6} \eta(4 z)^{6} \eta(16 z)^{6}}{\eta(2 z)^{3} \eta(8 z)^{3}}=q^{4} \prod_{n=1}^{\infty} \frac{\left(1-q^{n}\right)^{6}\left(1-q^{4 n}\right)^{6}\left(1-q^{16 n}\right)^{6}}{\left(1-q^{2 n}\right)^{3}\left(1-q^{8 n}\right)^{3}}=\sum_{n=1}^{\infty} t_{4}(n) q^{n}, \\
& \frac{\eta(z)^{4} \eta(4 z)^{6} \eta(16 z)^{8}}{\eta(2 z)^{2} \eta(8 z)^{4}}=q^{5} \prod_{n=1}^{\infty} \frac{\left(1-q^{n}\right)^{4}\left(1-q^{4 n}\right)^{6}\left(1-q^{16 n}\right)^{8}}{\left(1-q^{2 n}\right)^{2}\left(1-q^{8 n}\right)^{4}}=\sum_{n=1}^{\infty} t_{5}(n) q^{n}, \\
& \frac{\eta(z)^{2} \eta(4 z)^{6} \eta(16 z)^{10}}{\eta(2 z) \eta(8 z)^{5}}=q^{6} \prod_{n=1}^{\infty} \frac{\left(1-q^{n}\right)^{2}\left(1-q^{4 n}\right)^{6}\left(1-q^{16 n}\right)^{10}}{\left(1-q^{2 n}\right)\left(1-q^{8 n}\right)^{5}}=\sum_{n=1}^{\infty} t_{6}(n) q^{n}, \\
& \frac{\eta(4 z)^{6} \eta(16 z)^{12}}{\eta(8 z)^{6}}=q^{7} \prod_{n=1}^{\infty} \frac{\left(1-q^{4 n}\right)^{6}\left(1-q^{16 n}\right)^{12}}{\left(1-q^{8 n}\right)^{6}}=\sum_{n=1}^{\infty} t_{7}(n) q^{n} .
\end{aligned}
$$

By the definition of $T(n)$ the generating function $\sum_{n=1}^{\infty} T(n) q^{n}$ is in $S_{6}\left(\Gamma_{0}(16)\right)$. The space of cusp forms breaks into the spaces of newforms and oldforms. We write

$$
S_{k}\left(\Gamma_{0}(N)\right)=S_{k}^{\text {new }}\left(\Gamma_{0}(N)\right) \oplus S_{k}^{\text {old }}\left(\Gamma_{0}(N)\right) .
$$

By [15, Theorem 2.27], if $h(q)=q+\sum_{n=2}^{\infty} a(n) q^{n} \in S_{k}^{\text {new }}\left(\Gamma_{0}(4 N)\right)$ is a normalized newform, then $a(2)=0$. Applying the Hecke operator $T_{2}$ to $h(q)$ (see [15, Definition 2.1]), we get

$$
T_{2}(h)(q)=\sum_{n=1}^{\infty} a(2 n) q^{n}=a(2) q+a(4) q^{2}+a(6) q^{3}+\cdots .
$$

Since newforms are eigenforms for all Hecke operators (see [8, \$4.3]), we have $T_{2}(h)=\lambda h$ for some constant $\lambda$, but since $a(2)=0, \lambda$ must be zero. So, $T_{2}(h)$ is identically zero, and thus $a(2 n)=0$ for $n=1,2, \ldots$ Thus all forms in $S_{k}^{\text {new }}\left(\Gamma_{0}(4)\right), S_{k}^{\text {new }}\left(\Gamma_{0}(8)\right)$, and $S_{k}^{\text {new }}\left(\Gamma_{0}(16)\right)$ have Fourier expansions of the form $a(1) q+a(3) q^{3}+a(5) q^{5}+\cdots$. In particular, $a(8 n)=0$. Now we consider the space $S_{k}^{\text {old }}\left(\Gamma_{0}(16)\right)$. For a modular form $h(q), V(d)$ is defined by $h(q) \mid V(d)=h\left(q^{d}\right)$. By $[15,(2.16)]$ we have

$$
S_{k}^{\text {old }}\left(\Gamma_{0}(N)\right)=\bigoplus_{\substack{d M \mid N \\ M \neq N}} S_{k}\left(\Gamma_{0}(M)\right) \mid V(d)
$$

Note that there are no forms in $S_{6}\left(\Gamma_{0}(1)\right)$ and $S_{6}\left(\Gamma_{0}(2)\right)$. Thus $S_{6}^{\text {old }}\left(\Gamma_{0}(16)\right)$ is obtained from $S_{6}\left(\Gamma_{0}(4)\right)$ and $S_{6}\left(\Gamma_{0}(8)\right)$. Indeed, $S_{6}\left(\Gamma_{0}(4)\right)=S_{6}^{\text {new }}\left(\Gamma_{0}(4)\right)$ and $S_{6}\left(\Gamma_{0}(8)\right)=S_{6}^{\text {new }}\left(\Gamma_{0}(8)\right) \oplus$ $S_{6}\left(\Gamma_{0}(4)\right) \oplus S_{6}\left(\Gamma_{0}(4)\right) \mid V(2)$. By the definition of the $V$-operators the space $S_{6}^{\text {old }}\left(\Gamma_{0}(16)\right)$ is spanned by forms

$$
\begin{aligned}
& b(1) q+b(3) q^{3}+b(5) q^{5}+\cdots=\sum_{n \equiv 1(\bmod 2)} b(n) q^{n}, \\
& c(2) q^{2}+c(6) q^{6}+c(10) q^{10}+\cdots=\sum_{n \equiv 2(\bmod 4)} c(n) q^{n}, \\
& d(4) q^{4}+d(12) q^{12}+d(20) q^{20}+\cdots=\sum_{n \equiv 4(\bmod 8)} d(n) q^{n} .
\end{aligned}
$$

We can observe that $b(n), c(n), d(n)=0$ for all $n \equiv 0(\bmod 8)$. Therefore, for any form in $S_{6}\left(\Gamma_{0}(16)\right)$, its $8 n$th coefficients vanish. In particular, $T(n)=0$ for all $n \equiv 0(\bmod 8)$. 
Proof Theorem 5 We consider $F(q)^{3} . F(q)^{3}=\sum_{m \geq 0} \mathfrak{a}_{3}(m) q^{m}$ is a modular form in $M_{6}\left(\Gamma_{0}(16)\right)$. This space is 13-dimensional, so $F(q)^{3}$ can be expressed as a linear combination of thirteen linearly independent modular forms in $M_{6}\left(\Gamma_{0}(16)\right)$. We take seven cusp forms $\sum_{n \geq 1} t_{i}(n) q^{n}$ for $i=1,2, \ldots, 7$ and choose

$$
\begin{aligned}
& E_{6}(q)=1-504 \sum_{n=1}^{\infty} \sigma_{5}(n) q^{n}, \quad E_{6}\left(q^{2}\right)=1-504 \sum_{n=1}^{\infty} \sigma_{5}\left(\frac{n}{2}\right) q^{n}, \\
& E_{6}\left(q^{4}\right)=1-504 \sum_{n=1}^{\infty} \sigma_{5}\left(\frac{n}{4}\right) q^{n}, \quad E_{6}\left(q^{8}\right)=1-504 \sum_{n=1}^{\infty} \sigma_{5}\left(\frac{n}{8}\right) q^{n}, \\
& E_{6}\left(q^{16}\right)=1-504 \sum_{n=1}^{\infty} \sigma_{5}\left(\frac{n}{16}\right) q^{n}, \quad \frac{i E_{6}(i q)-i E_{6}(-i q)}{1008}=\sum_{\substack{n=1 \\
n \text { odd }}}^{\infty}(-1)^{\frac{n-1}{2}} \sigma_{5}(n) q^{n}
\end{aligned}
$$

in the Eisenstein subspace. Then we obtain the following formula:

$$
\begin{aligned}
F(q)^{3}= & \frac{1}{63} E_{6}\left(q^{4}\right)-\frac{22}{21} E_{6}\left(q^{8}\right)+\frac{128}{63} E_{6}\left(q^{16}\right)+\frac{1}{4} \frac{i E_{6}(i q)-i E_{6}(-i q)}{1008} \\
& -\frac{49}{4} \sum_{n=1}^{\infty} t_{1}(n) q^{n}-99 \sum_{n=1}^{\infty} t_{2}(n) q^{n}-210 \sum_{n=1}^{\infty} t_{3}(n) q^{n}-80 \sum_{n=1}^{\infty} t_{4}(n) q^{n} \\
& -240 \sum_{n=1}^{\infty} t_{5}(n) q^{n}-384 \sum_{n=1}^{\infty} t_{6}(n) q^{n}-256 \sum_{n=1}^{\infty} t_{7}(n) q^{n} .
\end{aligned}
$$

Since

$$
T(n)=\frac{49}{256} t_{1}(n)+\frac{99}{64} t_{2}(n)+\frac{105}{32} t_{3}(n)+\frac{5}{4} t_{4}(n)+\frac{15}{4} t_{5}(n)+6 t_{6}(n)+4 t_{7}(n),
$$

we have

$$
\mathfrak{a}_{3}(n)= \begin{cases}\frac{1}{4}(-1)^{\frac{n-1}{2}} \sigma_{5}(n)-64 T(n) & \text { if } n \text { is odd } \\ -8 \sigma_{5}\left(\frac{n}{4}\right)+528 \sigma_{5}\left(\frac{n}{8}\right)-1024 \sigma_{5}\left(\frac{n}{16}\right)-64 T(n) & \text { if } n \neq 0 \text { is even, } \\ 1 & \text { if } n=0 .\end{cases}
$$

Note that

$$
\begin{aligned}
F(q)^{3} & =\sum_{n \geq 0} \mathfrak{a}_{3}(n) q^{n}=\left(\sum_{n \geq 0} \mathfrak{a}_{1}(n) q^{n}\right)^{3}=\sum_{n \geq 0} \sum_{a_{1}+a_{2}+a_{3}=n} \mathfrak{a}_{1}\left(a_{1}\right) \mathfrak{a}_{1}\left(a_{2}\right) \mathfrak{a}_{1}\left(a_{3}\right) q^{n} \\
& =-64 \sum_{n \geq 0} \sum_{a_{1}+a_{2}+a_{3}=n}\left(-\frac{1}{4}\right)^{\epsilon\left(a_{1}, a_{2}, a_{3}\right)} \bar{\sigma}\left(a_{1}\right) \bar{\sigma}\left(a_{2}\right) \bar{\sigma}\left(a_{3}\right) q^{n},
\end{aligned}
$$

where $\epsilon\left(a_{1}, a_{2}, a_{3}\right)=\#\left\{a_{i}=0 \mid i=1,2,3\right\}$. Combining this with the previous result, we get

$$
\begin{aligned}
& \sum_{a_{1}+a_{2}+a_{3}=n}\left(-\frac{1}{4}\right)^{\epsilon\left(a_{1}, a_{2}, a_{3}\right)} \bar{\sigma}\left(a_{1}\right) \bar{\sigma}\left(a_{2}\right) \bar{\sigma}\left(a_{3}\right) \\
& = \begin{cases}\frac{1}{256}(-1)^{\frac{n+1}{2}} \sigma_{5}(n)+T(n) & \text { if } n \text { is odd } \\
\frac{1}{8} \sigma_{5}\left(\frac{n}{4}\right)-\frac{33}{4} \sigma_{5}\left(\frac{n}{8}\right)+16 \sigma_{5}\left(\frac{n}{16}\right)+T(n) & \text { if } n \neq 0 \text { is even. }\end{cases}
\end{aligned}
$$


Observe that for $n \geq 3$,

$$
\begin{aligned}
& \sum_{a_{1}+a_{2}+a_{3}=n}\left(-\frac{1}{4}\right)^{\epsilon\left(a_{1}, a_{2}, a_{3}\right)} \bar{\sigma}\left(a_{1}\right) \bar{\sigma}\left(a_{2}\right) \bar{\sigma}\left(a_{3}\right) \\
& =\sum_{\substack{a_{1}+a_{2}+a_{3}=n \\
a_{1}, a_{2}, a_{3} \geq 1}} \bar{\sigma}\left(a_{1}\right) \bar{\sigma}\left(a_{2}\right) \bar{\sigma}\left(a_{3}\right)-\frac{3}{4} \sum_{k=1}^{n-1} \bar{\sigma}(k) \bar{\sigma}(n-k)+\frac{3}{16} \bar{\sigma}(n) \\
& =\sum_{\substack{a_{1}+a_{2}+a_{3}=n \\
a_{1}, a_{2}, a_{3} \geq 1}} \bar{\sigma}\left(a_{1}\right) \bar{\sigma}\left(a_{2}\right) \bar{\sigma}\left(a_{3}\right) \\
& \quad-\frac{3}{4} \sigma_{3}\left(\frac{n}{2}\right)+\frac{27}{2} \sigma_{3}\left(\frac{n}{4}\right)-24 \sigma_{3}\left(\frac{n}{8}\right)-\frac{3}{16} \bar{\sigma}(n)+\frac{3}{8} \mathfrak{e}_{1}(n),
\end{aligned}
$$

where the last equality holds by (52). Finally, we have

$$
\begin{aligned}
& \sum_{\substack{a_{1}+a_{2}+a_{3}=n \\
a_{1}, a_{2}, a_{3} \geq 1}} \bar{\sigma}\left(a_{1}\right) \bar{\sigma}\left(a_{2}\right) \bar{\sigma}\left(a_{3}\right) \\
& = \begin{cases}(-1)^{\frac{n+1}{2}}\left(\frac{1}{256} \sigma_{5}(n)-\frac{3}{16} \sigma(n)\right)+T(n)-\frac{3}{8} \mathfrak{e}_{1}(n) & \text { if } n \text { is odd } \\
\frac{3}{4} \sigma_{3}\left(\frac{n}{2}\right)+T(n) & \text { if } n \equiv 2(\bmod 4) \\
\frac{1}{8} \sigma_{5}\left(\frac{n}{4}\right)+\frac{3}{4} \sigma_{3}\left(\frac{n}{2}\right)-\frac{27}{2} \sigma_{3}\left(\frac{n}{4}\right)+\frac{3}{8} \sigma\left(\frac{n}{4}\right)+T(n) & \text { if } n \equiv 4(\bmod 8), \\
\frac{1}{8} \sigma_{5}\left(\frac{n}{4}\right)-\frac{33}{4} \sigma_{5}\left(\frac{n}{8}\right)+16 \sigma_{5}\left(\frac{n}{16}\right) & \\
\quad+\frac{3}{4} \sigma_{3}\left(\frac{n}{2}\right)-\frac{27}{2} \sigma_{3}\left(\frac{n}{4}\right)+24 \sigma_{3}\left(\frac{n}{8}\right)+\frac{3}{16} \bar{\sigma}(n) & \text { if } n \equiv 0(\bmod 8)\end{cases}
\end{aligned}
$$

Lemma 13 If $N$ is an integer, then $\sum_{m=1}^{N-1} \mathfrak{a}_{2}(8 m) \mathfrak{a}_{2}(8(N-m))=\frac{16}{255}\left(21,167 \sigma_{7}(2 N)-\right.$ $\left.1327 \sigma_{7}(N)-256 \sigma_{7}\left(\frac{N}{2}\right)-20,417 \sigma_{3}(2 N)+697 \sigma_{3}(N)+136 \sigma_{3}\left(\frac{N}{2}\right)+750 \mathfrak{d}(2 N)-2160 \mathfrak{d}(N)\right)$.

Proof Let

$$
\begin{array}{ll}
U_{1}:=\sum_{m=1}^{N-1} \sigma_{3}(2 m) \sigma_{3}(2(N-m)), & U_{2}:=\sum_{m=1}^{N-1} \sigma_{3}(2 m) \sigma_{3}(N-m), \\
U_{3}:=\sum_{m=1}^{N-1} \sigma_{3}(m) \sigma_{3}(2(N-m)), & U_{4}:=\sum_{m=1}^{N-1} \sigma_{3}(m) \sigma_{3}(N-m) .
\end{array}
$$

By Lemma 8 we obtain

$$
\begin{aligned}
& \sum_{m=1}^{N-1} \mathfrak{a}_{2}(8 m) \mathfrak{a}_{1}(8(N-m)) \\
& \quad=16^{2} \sum_{m=1}^{N-1}\left(\sigma_{3}(2 m)-25 \sigma_{3}(m)\right)\left(\sigma_{3}(2(N-m))-25 \sigma_{3}(N-m)\right) \\
& \quad=16^{2}\left(U_{1}-25 U_{2}-25 U_{3}+25^{2} U_{4}\right) .
\end{aligned}
$$

By (3) and Proposition 1 we deduce Lemma 13. 
Proof of Theorem 6 We can regard $F(q)^{4}$ as a modular form in the five-dimensional space $M_{8}\left(\Gamma_{0}(4)\right)$, which is spanned by $E_{8}(q), E_{8}\left(q^{2}\right), E_{8}\left(q^{4}\right), H(q)$, and $Y(q)^{2}$, where

$$
E_{8}(q)=1+480 \sum_{n=1}^{\infty} \sigma_{7}(n) q^{n}
$$

Then we get the following formula:

$$
F(q)^{4}=-\frac{1}{255} E_{8}\left(q^{2}\right)+\frac{256}{255} E_{8}\left(q^{4}\right)-16 H(q)-\frac{512}{17} Y(q)^{2} .
$$

Thus we have

$$
\mathfrak{a}_{4}(n)=\frac{16}{17}\left(-2 \sigma_{7}\left(\frac{n}{2}\right)+512 \sigma_{7}\left(\frac{n}{4}\right)-17 \mathfrak{d}(n)-32 \mathfrak{e}_{2}(n)\right)
$$

or, equivalently,

$$
\mathfrak{a}_{4}(n)=\frac{16}{17}\left(-2 \sigma_{7}\left(\frac{n}{2}\right)+512 \sigma_{7}\left(\frac{n}{4}\right)-17 \mathfrak{e}_{2}(2 n)-32 \mathfrak{e}_{2}(n)\right),
$$

since $H(q)=Y\left(q^{1 / 2}\right)^{2}$, so that $\mathfrak{d}(n)=\mathfrak{e}_{2}(2 n)$. In particular, if $n$ is odd, then $\mathfrak{a}_{4}(n)=-16 \mathfrak{d}(n)=$ $-16 \mathfrak{e}_{2}(2 n)$. Also, we get

$$
\begin{gathered}
\sum_{a_{1}+a_{2}+a_{3}+a_{4}=n}\left(-\frac{1}{4}\right)^{\epsilon\left(a_{1}, a_{2}, a_{3}, a_{4}\right)} \bar{\sigma}\left(a_{1}\right) \bar{\sigma}\left(a_{2}\right) \bar{\sigma}\left(a_{3}\right) \bar{\sigma}\left(a_{4}\right) q^{n} \\
=\frac{1}{272}\left(-2 \sigma_{7}\left(\frac{n}{2}\right)+512 \sigma_{7}\left(\frac{n}{4}\right)-17 \mathfrak{d}(n)-32 \mathfrak{e}_{2}(n)\right) .
\end{gathered}
$$

Observing that

$$
\begin{aligned}
& \sum_{a_{1}+a_{2}+a_{3}+a_{4}=n}\left(-\frac{1}{4}\right)^{\epsilon\left(a_{1}, a_{2}, a_{3}, a_{4}\right)} \bar{\sigma}\left(a_{1}\right) \bar{\sigma}\left(a_{2}\right) \bar{\sigma}\left(a_{3}\right) \bar{\sigma}\left(a_{4}\right) \\
& =\sum_{\substack{a_{1}+a_{2}+a_{3}+a_{4}=n \\
a_{1}, a_{2}, a_{3}, a_{4} \geq 1}} \bar{\sigma}\left(a_{1}\right) \bar{\sigma}\left(a_{2}\right) \bar{\sigma}\left(a_{3}\right) \bar{\sigma}\left(a_{4}\right)-\sum_{\substack{a_{1}+a_{2}+a_{3}=n \\
a_{1}, a_{2}, a_{3} \geq 1}} \bar{\sigma}\left(a_{1}\right) \bar{\sigma}\left(a_{2}\right) \bar{\sigma}\left(a_{3}\right) \\
& \quad+\frac{3}{8} \sum_{k=1}^{n-1} \bar{\sigma}(k) \bar{\sigma}(n-k)-\frac{1}{16} \bar{\sigma}(n),
\end{aligned}
$$


we obtain

$$
\begin{aligned}
& \sum_{\substack{a_{1}+a_{2}+a_{3}+a_{4}=n \\
a_{1}, a_{2}, a_{3}, a_{4} \geq 1}} \bar{\sigma}\left(a_{1}\right) \bar{\sigma}\left(a_{2}\right) \bar{\sigma}\left(a_{3}\right) \bar{\sigma}\left(a_{4}\right) \\
& = \begin{cases}(-1)^{\frac{n+1}{2}}\left(\frac{1}{256} \sigma_{5}(n)-\frac{1}{16} \sigma(n)\right)+T(n)-\frac{1}{16} \mathfrak{d}(n)-\frac{3}{16} \mathfrak{e}_{1}(n) & \text { if } n \text { is odd } \\
-\frac{1}{136} \sigma_{7}\left(\frac{n}{2}\right)+\frac{3}{8} \sigma_{3}\left(\frac{n}{2}\right)+T(n)-\frac{1}{16} \mathfrak{d}(n)-\frac{2}{17} \mathfrak{e}_{2}(n) & \text { if } n \equiv 2(\bmod 4), \\
-\frac{1}{136} \sigma_{7}\left(\frac{n}{2}\right)+\frac{32}{17} \sigma_{7}\left(\frac{n}{4}\right)+\frac{1}{8} \sigma_{5}\left(\frac{n}{4}\right)+\frac{3}{8} \sigma_{3}\left(\frac{n}{2}\right)-\frac{27}{4} \sigma_{3}\left(\frac{n}{4}\right) & \\
+\frac{1}{8} \sigma\left(\frac{n}{4}\right)+T(n)-\frac{1}{16} \mathfrak{d}(n)-\frac{2}{17} \mathfrak{e}_{2}(n) & \text { if } n \equiv 4(\bmod 8), \\
-\frac{1}{136} \sigma_{7}\left(\frac{n}{2}\right)+\frac{32}{17} \sigma_{7}\left(\frac{n}{4}\right)+\frac{1}{8} \sigma_{5}\left(\frac{n}{4}\right)-\frac{33}{4} \sigma_{5}\left(\frac{n}{8}\right) & \\
+16 \sigma_{5}\left(\frac{n}{16}\right)+\frac{3}{8} \sigma_{3}\left(\frac{n}{2}\right)-\frac{27}{4} \sigma_{3}\left(\frac{n}{4}\right)+12 \sigma_{3}\left(\frac{n}{8}\right)+\frac{1}{16} \bar{\sigma}(n) & \\
-\frac{1}{16} \mathfrak{d}(n)-\frac{2}{17} \mathfrak{e}_{2}(n) & \text { if } n \equiv 0(\bmod 8) .\end{cases}
\end{aligned}
$$

\section{Conclusion}

Although many other research papers about divisor functions, restricted divisor functions, and the coefficients of modular functions have been written in recent years, active, productive, and applied approaches are still continuing in these areas. For this reason, arithmetic properties for new identities of special numbers and polynomials involving eta quotients and modular forms and combinatorial numbers are constructed. By considering these coefficients with their modular equations, difference equations, and combinatorial equations, we obtained and studied various properties for divisor functions, restricted divisor functions, and some combinatorial numbers. The use of the convolution sums of these divisor functions is helpful in the theory of convolution sums of various restricted divisor functions and also helpful in theories of modular forms, elliptic curves, and partitions.

\section{Acknowledgements}

The corresponding author was supported by the Basic Science Research Program through the National Research Foundation of Korea (NRF) funded by the Ministry of Education (NRF-2018R1D1A1B07041132).

Funding

Not applicable.

Availability of data and materials

Not applicable.

\section{Competing interests}

The authors declare that they have no competing interests.

Authors' contributions

All the authors participated in every phase of the research conducted for this paper. All authors read and approved the final manuscript.

\section{Author details}

1 Department of Mathematics, Sungkyunkwan University, Suwon, South Korea. ${ }^{2}$ Department of Applied Mathematics, China Agricultural University, Beijing, China. ${ }^{3}$ Department of Mathematics and Institute of Pure and Applied

Mathematics, Jeonbuk National University, Jeonju, South Korea.

\section{Publisher's Note}

Springer Nature remains neutral with regard to jurisdictional claims in published maps and institutional affiliations. 


\section{References}

1. Besge, M.: Extrait d'une lettre de M. Besge à M. Liouville. J. Math. Pures Appl. 7256 (1862)

2. Cheng, N., Williams, K.S.: Evaluation of some convolution sums involving the sum of divisors functions. Yokohama Math. J. 52, 39-57 (2005)

3. Dickson, L.E.: History of the Theory of Numbers, Vol. I. Chelsea, New York (1952)

4. Fine, N.J.: Basic Hypergeometric Series and Applications. Am. Math. Soc., Providence (1988)

5. Glaisher, J.W.L.: On certain sums of products of quantities depending upon the divisors of a number. Mess. Math. 15 , $1-20(1885)$

6. Hua, L.-K.: Introduction to Number Theory. Bulletin of the London Mathematical Society, vol. 15 (1983)

7. Huard, J.G., Ou, Z.M., Spearman, B.K., Williams, K.S.: Elementary evaluation of certain convolution sums involving divisor functions. In: Number Theory for the Millennium, II, pp. 229-274 (2002)

8. Kilford, L.J.P.: Modular Forms, 2nd edn. Imperial College Press, London (2015)

9. Kim, A., Kim, D., Li, Y.: Convolution sums arising from divisor functions. J. Korean Math. Soc. 50, 331-360 (2013)

10. Kim, D., Bayad, A.: Convolution identities for twisted Eisenstein series and twisted divisor functions. Fixed Point Theory Appl. 2013, 81 (2013) 1-23

11. Lahiri, D.B.: On Ramanujan's function $\tau(n)$ and the divisor function $\sigma(n)$, I. Bull. Calcutta Math. Soc. 38, 193-206 (1946)

12. Lehmer, D.H.: Some functions of Ramanujan. Math. Stud. 27, 105-116 (1959)

13. Melfi, G.: Some problems in elementary number theory and modular forms. Ph.D. Thesis, University of Pisa (1997)

14. Melfi, G.: On some modular identities. In: Number Theory, Diophantine, Computational and Algebraic Aspects: Proceedings of the International Conference Held in Eger, Hungrary, pp. 371-382. de Gruyter, Berlin (1998)

15. Ono, K.: The Web of Modularity: Arithmetic of the Coefficients of Modular Forms and $q$-Series. CBMS Regional Conference Series in Mathematics, vol. 102 (2004)

16. Park, Y.: Evaluation of the convolution sums $\sum_{a k+b l+c m=n} \sigma(k) \sigma(i) \sigma(m)$ with $\operatorname{lcm}(a, b, c)=7,8$ or 9 . Int. J. Number Theory 14(6), 1637-1650 (2018)

17. Pehlivan, L., Williams, K.S.: The power series expansion of certain infinite products $q^{r} \prod_{n=1}^{\infty}\left(1-q^{n}\right)^{a_{1}}\left(1-q^{2 n}\right)^{a_{2}} \ldots\left(1-q^{m n}\right)^{a_{m}}$. Ramanujan J. 33, 23-53 (2014)

18. Ramanujan, S.: On certain trigonometric sums and their applications in the theory of numbers. Trans. Cambridge Philos. Soc. 22(15), 259-276 (1918) (pp. 179-199 of his Collected Papers)

19. Skoruppa, N.-P.: A quick combinatorial proof of Eisenstein series identities. J. Number Theory 43, 68-73 (1993)

20. Williams, K.S.: Number Theory in the Spirit of Liouville. Cambridge University Press, New York (2011)

21. Williams, K.S.: Fourier series of a class of eta quotients. Int. J. Number Theory 8(4), 993-1004 (2012)

\section{Submit your manuscript to a SpringerOpen ${ }^{\circ}$ journal and benefit from:}

- Convenient online submission

- Rigorous peer review

- Open access: articles freely available online

- High visibility within the field

- Retaining the copyright to your article

Submit your next manuscript at $>$ springeropen.com 